\title{
Relationship between Refraction and Prevalent as well as Incident Age-Related Maculopathy: The Rotterdam Study
}

\author{
M. Kamran Ikram, ${ }^{1}$ Redmer van Leeuwen, ${ }^{1}$ Jobannes $R$. Vingerling, ${ }^{1,2}$ Albert Hofman, ${ }^{1}$ \\ and Paulus T. V. M. de Jong ${ }^{1,3,4}$
}

Purpose. To study the relationship between baseline spherical equivalents (SphE) of refraction and prevalent as well as incident age-related maculopathy (pARM and iARM, respectively).

Methods. The study was performed as part of the Rotterdam Study, a population-based, prospective cohort study. The SphE (in diopters), measured with autorefraction and subjective optimization, was recorded in 6209 subjects aged 55 years or more. Aphakic or pseudophakic eyes at baseline were excluded. Stereoscopic transparencies of the macular region were graded according to the International Classification and Grading System. ARM was defined as large soft drusen with pigmentary changes, or indistinct drusen, or atrophic or neovascular age-related macular degeneration (AMD). For the prevalence analyses, ARM was classified into no, p(early)ARM, or pAMD, and in each subject the eye with the most advanced $\mathrm{ARM}$ and the corresponding refraction was selected. After a mean 5.2 years of follow-up, 4935 subjects had complete data for these incidence analyses. In each subject, the eye with iARM was selected.

Results. The age- and gender-adjusted odds ratio (OR) of pARM $(n=536)$ for every diopter of progress toward hyperopia was 1.09 (95\% confidence interval [CI]1.04-1.13). For p(early)ARM $(n=440)$ the OR was $1.09(1.04-1.14)$ and for PAMD $(n=96)$ the OR was $1.09(1.00-1.19)$. Baseline refraction was significantly associated with increased risk of iARM $(n=497)$. For each diopter of progress toward hyperopia the OR was 1.05 (95\% CI 1.01-1.10). Additional adjustments for smoking, atherosclerosis, and blood pressure did not alter the relationship.

From the Departments of ${ }^{1}$ Epidemiology and Biostatistics and ${ }^{2}$ Ophthalmology, Erasmus Medical Center, Rotterdam, The Netherlands; ${ }^{3}$ The Netherlands Ophthalmic Research Institute, Royal Academy of Arts and Sciences (KNAW), Amsterdam, The Netherlands; and ${ }^{4}$ Department of Ophthalmology, Academic Medical Center, Amsterdam, The Netherlands.

Presented in part at the annual meeting of the Association for Research in Vision and Ophthalmology, Fort Lauderdale, Florida, May 2003.

Supported by the Optimix Foundation, Amsterdam; the Netherlands Organization for Scientific Research (NWO), The Hague; Topcon Europe BV, Capelle a/d IJssel; The Netherlands Society for the Prevention of Blindness, Doorn; Blindenhulp Foundation, The Hague; Rotterdamse Blindenbelangen Foundation, Rotterdam; OOG Foundation, The Hague; The Edward and Marianne Blaauw Foundation, Amsterdam, The Netherlands.

Submitted for publication February 5, 2003; revised May 19, 2003; accepted May 29, 2003.

Disclosure: M.K. Ikram, None; R. van Leeuwen, None; J.R. Vingerling, None; A. Hofman, None; P.T.V.M. de Jong, None

The publication costs of this article were defrayed in part by page charge payment. This article must therefore be marked "advertisement" in accordance with 18 U.S.C. $\$ 1734$ solely to indicate this fact

Corresponding author: Paulus T. V. M. de Jong, The Netherlands Ophthalmic Research Institute, Meibergdreef 47, 1105 BA Amsterdam, The Netherlands; p.dejong@ioi.knaw.nl.
Conclusions. These population-based incidence data confirm results from prevalence and case-control studies that there is an association between hyperopia and ARM. (Invest Ophthalmol Vis Sci. 2003;44:3778-3782) DOI:10.1167/iovs.03-0120

A ge-related maculopathy (ARM) may be characterized by an accumulation of abnormal extracellular deposits (called drusen) in the vicinity of Bruch's membrane, ${ }^{1}$ with or without pigmentary changes at the level of the retinal pigment epithelium (RPE). Its end stage, also called age-related macular degeneration (AMD), is the major cause of incurable visual impairment in the Western world. Over the years, it has been proposed that these drusen are a manifestation of dysfunction and degeneration of the RPE and retina. ${ }^{1}$ These age-related changes may also be a manifestation of restricted exchange of nutrients and other metabolic products between the neural retina across Bruch's membrane toward the choroid.

At the moment, there is limited long-term effective treatment for AMD. ${ }^{2-4}$ In a small number of subjects with neovascular AMD, laser photocoagulation and photodynamic therapy can be successfully used in delaying visual loss. Recently, the AREDS trial ${ }^{5}$ showed a protective effect of high-dose supplementation with antioxidant vitamins and zinc in a subset of ARM cases. In view of preventive measures, better knowledge of pathophysiology and detection of early stages ${ }^{6}$ is important.

Different studies ${ }^{7}$ have looked at various risk factors involved in AMD, such as smoking, atherosclerosis, and genetic factors. Similarly, it has been hypothesized that ocular factors, such as cataract (extraction), iris color, and refractive errors may also be involved in the development of this disease. ${ }^{8,9}$ The association between hyperopia and AMD was first described by Maltzman et al. ${ }^{10}$ in 1979 in a case-control setting. After that, a few other case-control ${ }^{11-14}$ studies on this topic showed conflicting results. One report of a population-based crosssectional study ${ }^{15}$ mentioned a weak association between hyperopia and early ARM (per diopter of progress toward hyperopia: OR: 1.1 ; 95\% CI: 1.0-1.2). More recently, a populationbased follow-up study ${ }^{16}$ showed no relationship between refractive error and 10-year incidence of early ARM (hyperopia $>1.0$ D versus emmetropia: relative risk [RR]: 0.9: 95\% CI: 0.7-1.1) and AMD (RR: 1.2; 95\% CI: 0.6-2.3).

Because the nature of the association between refraction and ARM is still unclear and may provide insight into the pathogenesis of this disease, we examined the association between baseline refraction and prevalent (pARM) as well as incident ARM (iARM) in a population-based setting.

\section{Methods AND Materials}

\section{Population}

Information on the identification and description of the baseline study population has appeared in previous reports. ${ }^{17}$ Briefly, the Rotterdam Study is a population-based prospective cohort study of the frequency and determinants of common cardiovascular, locomotor, neurologic, 
TABLE 1. Baseline Characteristics and Prevalence of Early ARM and AMD per Stratum of Refraction

\begin{tabular}{|c|c|c|c|c|c|}
\hline & Advanced Myopia* & Myopiat & Emmetropia & Hyperopiaฐ & Advanced Hyperopial \\
\hline Number & 443 & 770 & 966 & 3180 & 850 \\
\hline Mean age $(y)$ & 67.4 & 68.0 & 67.2 & 68.6 & 71.9 \\
\hline Gender ( $\%$ female) & 53.3 & 56.1 & 58.9 & 59.6 & 63.1 \\
\hline Mean SphE (D) & -5.5 & -1.4 & 0.0 & +1.6 & +4.3 \\
\hline $\mathrm{p}($ early)ARM $(n, \%)$ & $16(3.6)$ & $57(7.4)$ & $50(5.2)$ & $227(7.1)$ & $90(10.6)$ \\
\hline pAMD $(n, \%)$ & $4(0.9)$ & $10(1.3)$ & $16(1.7)$ & $43(1.4)$ & $23(2.7)$ \\
\hline
\end{tabular}

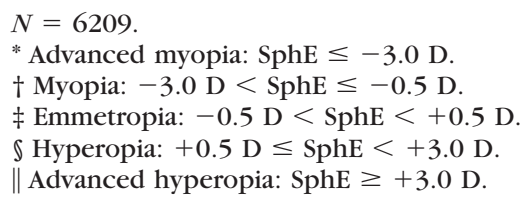

and ophthalmologic diseases. The eligible population $(n=10,275)$ consisted of all inhabitants aged 55 years or more in a suburb of Rotterdam, the Netherlands. Of these, 7983 (78\%) agreed to participate in the study. Because the ophthalmic phase of the study became operational after the screening of participants had started, a smaller portion $(n=6780)$ underwent ophthalmic examination. The study was conducted according to the tenets of the Declaration of Helsinki, and the medical ethics committee of the Erasmus Medical Center approved the study protocol. Written informed consent was obtained from all participants. Baseline interviews and examinations were performed from 1990 to mid-1993, followed by a first follow-up examination from 1993 to 1994 . A second follow-up screening took place from mid-1997 to the end of 1999 .

\section{Diagnosis of Age-Related Maculopathy}

A detailed description of the diagnostic procedures has been presented elsewhere ${ }^{18}$ Participants underwent a full eye examination, including stereo $35^{\circ}$ fundus photography (Topcon TRV-50VT fundus camera; Topcon Optical Company, Tokyo, Japan) centered on field 2 (the fovea) after pharmacologic mydriasis. The resultant transparencies were graded with $12.5 \times$ magnification according to the International Classification and Grading System for ARM and AMD. ${ }^{19}$ In this system, all ARM fundus signs within a standard circular area (diameter 6000 $\mu \mathrm{m})$ around the fovea are recorded. Two graders, trained according to the Wisconsin ARM grading system and each having 8 years experience, first graded the follow-up transparencies after which these were compared with those taken at baseline. The grading procedures and definitions, as well as the graders, were identical at baseline and at follow-up. Consensus sessions and between-grader comparisons were performed regularly. Weighted $\kappa$ statistics were 0.72 for soft distinct drusen, 0.80 for hyperpigmentation, and 0.58 for hypopigmentation.

ARM was defined as the presence of large ( $\geq 63 \mu \mathrm{m})$, soft, distinct drusen with pigmentary irregularities, indistinct $(\geq 125 \mu \mathrm{m})$ or reticular drusen, or atrophic or neovascular AMD. Atrophic AMD was defined as any sharply demarcated round or oval area of apparent absence of RPE, larger than $175 \mu \mathrm{m}$, irrespective of distance from the foveola but within the grid, with visible choroidal vessels and no neovascular AMD. Neovascular AMD was defined as the presence of a serous or hemorrhagic neuroretinal or RPE detachment and/or a subretinal neovascular membrane and/or a subretinal hemorrhage and/or a periretinal fibrous scar. Lesions that were considered to be the result of generalized disease, such as diabetic retinopathy, chorioretinitis, high myopia, trauma, congenital diseases, or photocoagulation for reasons other than neovascular AMD, were excluded from ARM classification.

\section{Refraction}

Refraction of each eye was taken as the mean of three measurements per eye with an autorefractometer (Topcon RM-2000; Topcon Corp., Tokyo, Japan) followed by subjective optimization. The medical doc- tors performing these measurements were trained by ophthalmologists, and quality control sessions were conducted routinely. In $2.5 \%$ of all subjects ( $1.9 \%$ of both eyes and $0.6 \%$ one eye) these autorefractometer measurements could not be obtained, mostly because of lens opacities or physiological miosis. The amount of correction of the subjects' spectacles (if present) was measured with a lensometer (Topcon CL-1000; Topcon Corp.). Subjects with no autorefraction data and who did not have glasses were excluded from our analyses. For all analyses, the spherical equivalents (SphE) were calculated and expressed in diopters.

\section{Study Sample}

Of the 6780 participants in the ophthalmic part of the baseline study, 6477 (95.5\%) underwent fundus photography, and 6418 (94.7\%) had gradable fundus transparencies in at least one eye. Prevalent ARM was diagnosed in 582 (9.1\%) subjects, including 106 with AMD. This resulted in a cohort of 5836 subjects at risk who were free of ARM (i.e., subjects with no drusen, only hard drusen or soft drusen, or pigmentary abnormalities only). Of this cohort, 283 (4.8\%) subjects died before the first follow-up examination, and another 789 (13.5\%) subjects died before the second follow-up. Of those alive at the first screening ( $n=5553)$, 46 subjects were lost to follow-up, 905 refused to participate, and 13 had ungradable photographs. Of those alive at the second follow-up ( $n=4764), 15$ subjects were lost to follow-up, 1267 refused to participate, and 47 had ungradable photographs. In total, 4822 subjects ( $83 \%$ of those at risk) participated in at least one follow-up examination.

Of the 6418 (94.7\%) subjects at baseline, 6209 (91.6\%) were included in the cross-sectional analysis, after excluding those with missing data on refraction and those who had had a cataract extraction in both eyes at baseline. In these subjects, prevalent early ARM (p(early)ARM) was diagnosed in 440 and prevalent AMD (pAMD) in 96 (total prevalent ARM (pARM) $n=536$ ). P(early)ARM was defined as pARM excluding PAMD

In the follow-up analyses of incident ARM (iARM), all persons free of ARM at baseline and who participated at least in one follow-up examination were included $(n=4822)$. Furthermore, if the second eye of a pARM case was free of ARM, that eye was also included in this analysis, because we were looking at an eye-specific risk factor. Hence, $4935(72.8 \%)$ subjects of the 6209 participants at baseline on whom we had complete data were included in the follow-up analyses, resulting in 497 cases of iARM. Incidence of ARM was defined as absence of ARM in an eye at baseline and presence of ARM in the same eye at follow-up. The mean follow-up time for the first examination was 2 years and for the second examination 6.5 years. The overall mean follow-up time was 5.2 years.

\section{Statistical Analyses}

Analyses were started by taking the SphE in diopters as a continuous variable. Next, cutoff points were taken to define (advanced) myopia 


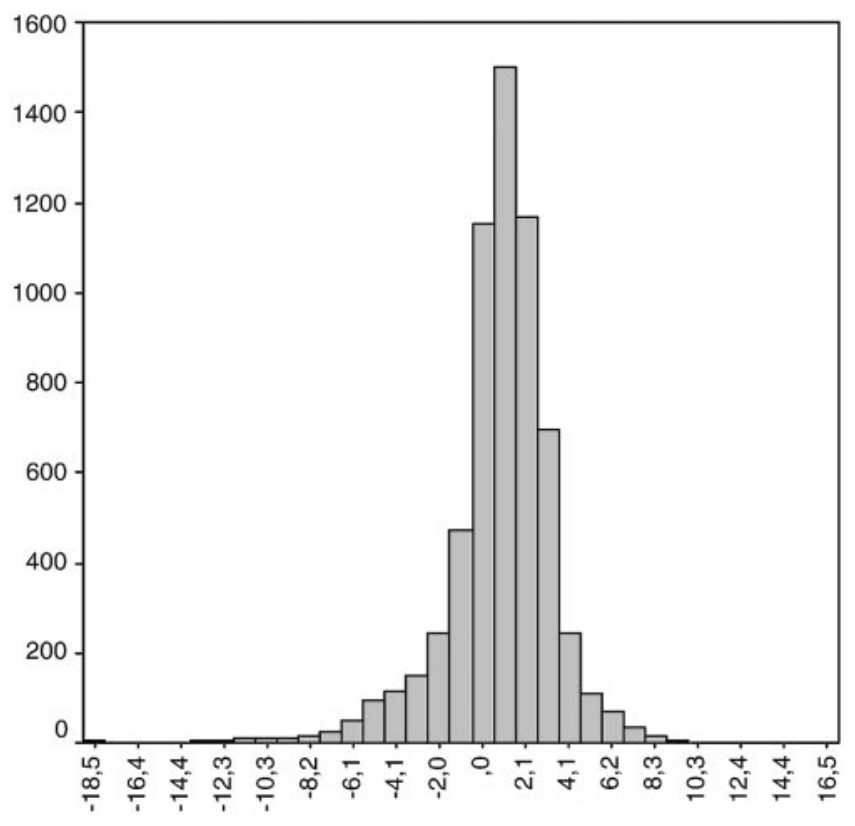

FigURE 1. Distribution of refraction at baseline $(n=6209)$.

and (advanced) hyperopia. In the legend of Table 1, the cutoff points are further specified. For categorized analyses, emmetropic eyes were used as the reference group.

For cross-sectional analyses in each subject, the eyes were classified into no ARM, p(early)ARM, or pAMD. Subsequently, the eye with most advanced ARM was chosen, and the corresponding refraction of the same eye was included in the analyses. If both eyes had no ARM or the same PARM diagnosis, the right eye was chosen. However, if one eye was aphakic or pseudophakic and the other phakic, the latter was included. Subjects with bilateral cataract extraction at baseline were excluded from the analyses. The association between the SphE (in a continuous and a categorized way) and pARM was analyzed using logistic regression models adjusted for age and gender. To explore this relationship further, we performed the analyses for p(early)ARM and pAMD separately.

For the incidence analyses, the eye that had iARM was selected, and the corresponding refraction of that same eye at baseline was used. In case iARM developed in both eyes, the right eye was chosen. Again, logistic regression modeling was performed to establish the relationship of baseline SphE with iARM correcting for age, gender, and follow-up time. Follow-up time was calculated using the dates on which the baseline and follow-up photographs were made. Because of the low number of incident AMD (iAMD) cases, the analyses were not performed separately for incident early ARM (i(early)ARM) and iAMD. In multivariable models, we further adjusted for smoking, atherosclerosis, and blood pressure at baseline.

\section{Results}

Table 1 shows some general characteristics and the prevalence of ARM in our study population that was used for the crosssectional analyses. In Figure 1 the distribution of refraction at baseline is presented (mean $\mathrm{SphE}=+0.83 \pm 2.6 \mathrm{D}$; [SD]). Analyses with SphE as a continuous variable showed that every diopter of progress toward hyperopia gave an age- and genderadjusted odds ratio (OR) of 1.09 (95\% confidence interval [CI]: 1.04-1.13) for pARM (Table 2). Furthermore, when p(early)ARM and PAMD were studied separately, the risk estimates were the same for both. Every diopter of progress toward hyperopia gave a significantly increased odds ratio for both p(early)ARM (OR: 1.09; 95\% CI: 1.04-1.14) and pAMD (OR: 1.09; 95\% CI: 1.00-1.19). Repeating the analyses with categorized SphEs (as defined in the legend to Table 1) showed that the risk of pARM was $46 \%$ higher in eyes with advanced hyperopia than in emmetropic eyes. In the categorized analyses, the association between advanced hyperopia and p(early)ARM (OR: 1.62; 95\% CI: 1.12-2.34) was still statistically significant, whereas the association disappeared for pAMD (OR: 1.14; 95\% CI: 0.57-2.24). After additional adjustments for smoking, atherosclerosis and blood pressure, a statistically significant risk of pARM (OR: 1.07; 95\% CI: 1.03-1.12) was found for every diopter of progress toward hyperopia.

There were 497 subjects with iARM, and Table 3 shows the incidence of ARM stratified according to the refractive status of the eye. The same, but attenuated, results were found for the association between baseline refraction and iARM (Table 4). After adjusting for age, gender, and follow-up time, every diopter of progress toward hyperopia increased the risk of iARM with 5\% (OR: 1.05; 95\% CI: 1.01-1.10). In the categorized analyses the risk estimates were not statistically significant, though there seemed to be a trend. After additional adjustments for smoking, atherosclerosis, and blood pressure, the risk of iARM (OR: 1.04; 95\% CI: 1.00-1.09) for every diopter of progress toward hyperopia remained statistically significant.

\section{Discussion}

Both the cross-sectional, and to a lesser extent, the follow-up results show that hyperopia is positively associated with ARM. For the proper interpretation of these findings we have to keep in mind several methodological aspects.

Subjects included in the present analyses differed from those excluded at baseline. These excluded subjects were not only those who did not participate in this study in the first place, but also those who were excluded because of missing data, ungradable photographs at baseline, or bilateral cataract extraction at baseline. Subjects who had had a bilateral cataract extraction were excluded, because we did not know the true refractive value of their natural lens and because of a potential

TABLE 2. Odds Ratios of Prevalent ARM According to Spherical Equivalents of Refraction

\begin{tabular}{lccccc}
\hline & $\begin{array}{c}\text { SphE } \\
\text { (Continuous)* } \\
\text { OR (95\% CI) }\end{array}$ & $\begin{array}{c}\text { Advanced } \\
\text { Myopia } \\
\text { vs. } \\
\text { Emmetropia } \\
\text { OR (95\% CI) }\end{array}$ & $\begin{array}{c}\text { Myopia vs. } \\
\text { Emmetropia } \\
\text { OR (95\% CI) }\end{array}$ & $\begin{array}{c}\text { Hyperopia vs. } \\
\text { Emmetropia } \\
\text { OR (95\% CI) }\end{array}$ & $\begin{array}{c}\text { Advanced Hyperopia vs. } \\
\text { Emmetropia } \\
\text { OR (95\% CI) }\end{array}$ \\
\hline pARM $(n=536)$ & $1.09(1.04-1.13)$ & $0.64(0.38-1.09)$ & $1.21(0.83-1.74)$ & $1.18(0.89-1.58)$ & $1.46(1.05-2.04)$ \\
p(early)ARM $(n=440)$ & $1.09(1.04-1.14)$ & $0.67(0.37-1.20)$ & $1.37(0.92-2.05)$ & $1.32(0.96-1.82)$ & $1.62(1.12-2.34)$ \\
pAMD $(n=96)$ & $1.09(1.00-1.19)$ & $0.61(0.20-1.89)$ & $0.71(0.31-1.64)$ & $0.87(0.47-1.59)$ & $1.14(0.57-2.24)$ \\
\hline
\end{tabular}

Data adjusted for age and gender. For definition of conditions, see Table 1.

* Range, $-18.75 \mathrm{D}$ to $+15.13 \mathrm{D}$; OR for every diopter of progress toward hyperopia. 
TABLE 3. Incidence of ARM per Stratum of Refraction in Absolute Numbers

\begin{tabular}{lccccc}
\hline & Advanced Myopia & Myopia & Emmetropia & Hyperopia & Advanced Hyperopia \\
\hline Number & 372 & 622 & 778 & 623 & 2540 \\
iARM* $(n, \%)$ & $26(7.0)$ & $48(7.7)$ & $73(9.4)$ & $270(10.6)$ & $80(12.8)$ \\
\hline
\end{tabular}

For definition of conditions, see Table 1. $n=4935$.

* Due to the low number of iAMD cases, they are not presented separately.

relationship between cataract extraction and ARM. ${ }^{9}$ To avoid misclassification of refractive status, these subjects had to be excluded. The excluded subjects at baseline were on average older and more often institutionalized. Exclusion of this older cohort, that probably contained relatively more cases of ARM due to the older age distribution, may cause an imprecision in the estimate of the associations, leading to wider confidence intervals. However, we do not think that the point estimates (OR) were affected due to selection bias by this exclusion. Although it is possible that having ARM causes nonparticipation, it is in our opinion unlikely that this nonparticipation was influenced by the refractive status.

This issue becomes even more important regarding the iARM analyses. As in any follow-up cohort, ours also showed that a relatively healthier population visited the research center during the follow-up examinations, which again produced an imprecision of an underlying association, but probably not a selection bias.

Taking SphE as a continuous variable assumes that there is no biological difference between myopia and hyperopia. Because this assumption is not based on any empiric evidence and because there are many different ocular disorders associated with myopia versus hyperopia, we preferred to define myopia and hyperopia also using cutoff values, with a group of emmetropic eyes as reference category. After doing so, we can still conclude that the relationship between ARM and hyperopia seems to be unaltered. Furthermore, the cutoff SphEs were chosen after considering the distribution of refraction in this population. When the analyses were repeated with other cutoffs, we saw similar results (data not shown). We decided to take the presented cutoff values mainly to secure large enough numbers in all categories.

Our cross-sectional data have limitations when it comes to causal relationships. However, because nearly all previous studies have used cross-sectional data for this association, we also analyzed our p(early)ARM and pAMD cases to compare our results with those in other studies. One report from a population-based cross-sectional study mentioned a weak association between hyperopia and only early $\mathrm{ARM}^{15}$ (per diopter of progress toward hyperopia; OR: $1.1,95 \% \mathrm{CI}$ : $1.0-1.2)$. The case-control AREDS study ${ }^{13}$ showed an association between hyperopia and large drusen as well as with neovascular and atrophic AMD. However, recently a population-based follow-up study ${ }^{16}$ showed no relationship between refractive status and the 10-year incidence of ARM. On the basis of our cross-sectional analyses, we can confirm that there seems to be a relationship between SphE and p(early)ARM and pAMD.
Furthermore, our results from the follow-up analyses support the hypothesis that there may be a causal relationship. In our attempt to fully explore the relationship between refraction and i(early)ARM as well as iAMD separately we were, however, limited by the small number of iAMD cases

Also, diagnostic procedures must be considered. Drusen in myopic fundi may be more difficult to assess because the usually fairer RPE and choroidal pigmentation results in a blonder fundus and less contrast. Another diagnostic pitfall may be that during the grading of the fundus transparencies no Littmann's correction was used for the variation in magnification caused by the refractive state of that eye. A photograph of a hyperopic eye will lead to a larger magnification due to the hyperopia compared with a myopic (or even an emmetropic) eye. This could have introduced a misclassification into our grading of drusen and may play a role to a certain extent in the cross-sectional analyses. In the follow-up analyses, however, the incident cases were defined as a change in ARM stage and the absolute dimensions of the drusen per se were not important. Thus, here the magnification should pose fewer problems. There could finally be a tendency to classify a "neovascular AMD" eye in a myopic fundus with a few or no drusen as a myopic disciform reaction or Fuch's spot instead of neovascular AMD. This could (partly) account for the association we found between hyperopia and neovascular pAMD (per diopter of progress toward hyperopia: OR: 1.21, 95\% CI: 1.09-1.36).

Additional adjustments for other known risk factors such as smoking, blood pressure, and atherosclerosis did not significantly alter this relationship, showing that refraction has an additional effect on the development of ARM. At baseline $13.4 \%$ of participants used any type of micronutrient supplements, but we did not have any information on the exact dose, type, and duration of use. Moreover, we are unaware of an association between micronutrient use and refraction, therefore we did not adjust for this use.

The pathophysiological mechanism by which hyperopia may lead to ARM still remains to be elucidated. We think that of the three components determining the refraction of an eye, corneal curvature, lens power, and axial length, the latter one is most likely to play a role in the pathogenesis of ARM. In general, hyperopic eyes are smaller and have thicker and more rigid and compact sclerae. ${ }^{20}$ This generalized stiffness of the sclera may cause an increase in resistance of the choroidal venous outflow ${ }^{21,22}$ with throttling of the vorticose veins, and a thicker choroid. ${ }^{23,24}$ Both histologic and in vivo studies with laser Doppler flow measurements have shown an increased choroidal resistance in AMD-cases compared with gender- and

TABLE 4. Odds Ratios of Early Incident ARM, According to Spherical Equivalents of Refraction

\begin{tabular}{lccccc}
\hline & $\begin{array}{c}\text { SphE } \\
\text { (Continuous)* } \\
\text { OR (95\% CI) }\end{array}$ & $\begin{array}{c}\text { Advanced Myopia } \\
\text { vs. Emmetropia } \\
\text { OR (95\% CI) }\end{array}$ & $\begin{array}{c}\text { Myopia vs. } \\
\text { Emmetropia } \\
\text { OR (95\% CI) }\end{array}$ & $\begin{array}{c}\text { Hyperopia vs. } \\
\text { Emmetropia } \\
\text { OR (95\% CI) }\end{array}$ & $\begin{array}{c}\text { Advanced Hyperopia } \\
\text { vs. Emmetropia } \\
\text { OR (95\% CI) }\end{array}$ \\
\hline iARM $(n=497)$ & $1.05(1.01-1.10)$ & $0.69(0.43-1.11)$ & $0.79(0.53-1.16)$ & $1.09(0.82-1.43)$ & $1.20(0.85-1.69)$ \\
\hline
\end{tabular}

Data adjusted for age, gender and follow-up time. For definition of conditions, see Table 1.

* Range, $-18.75 \mathrm{D}$ to $+9.63 \mathrm{D}$; OR for every diopter of progress toward hyperopia. 
age-matched controls. ${ }^{25}$ We speculate that decreased flow prevents easy exchange of nutrients and metabolic products across the RPE and results in drusen formation and thickening of Bruch's membrane. However, the exact role of these vascular abnormalities ${ }^{26}$ in the pathogenesis of ARM remains unclear.

Other speculations that may explain the observed association with hyperopia are that poorer cooling of the retina by an impaired choroidal blood flow may lead to a higher susceptibility to oxidative stress. Also the thicker retina in hyperopic versus myopic eyes may have a higher need for oxygen and nutrients. Furthermore, it is uncertain whether the photoreceptor density per square millimeter of the fovea or per RPE cell is different in hyperopia.

Although the magnitude of the risk estimates associated with refraction is lower than that of the major well-known risk factors such as age and smoking, ${ }^{7}$ the risk of $5 \%$ to $9 \%$ per diopter of progress toward hyperopia is still a considerable increase. In view of these findings, special attention may have to be given to older persons with hyperopia (e.g., over 70 years with hyperopia $>3.0$ diopter) to offer them in the presence of ARM a potential benefit from micronutrient supplementation.

In conclusion, this large population-based cohort study showed also in a prospective way that hyperopia is a risk factor for ARM.

\section{References}

1. Mullins RF, Russell SR, Anderson DH, Hageman GS. Drusen associated with aging and age-related macular degeneration contain proteins common to extracellular deposits associated with atherosclerosis, elastosis, amyloidosis, and dense deposit disease. FASEB J. 2000; $14: 835-846$.

2. Macular Photocoagulation Study Group. Argon laser photocoagulation for neovascular maculopathy: five-year results from randomized clinical trials. Arch Ophthalmol. 1991;109:1109-1114.

3. Moisseiev J, Alhalel A, Masuri R, Treister G. The impact of the macular photocoagulation study results on the treatment of exudative age-related macular degeneration. Arch Ophthalmol. 1995; 113:185-189.

4. Bressler NM. Photodynamic therapy of subfoveal choroidal neovascularization in age-related macular degeneration with verteporfin: two-year results of 2 randomized clinical trials-tap report 2. Arch Ophthalmol. 2001;119:198-207.

5. A randomized, placebo-controlled, clinical trial of high-dose supplementation with vitamins $\mathrm{C}$ and $\mathrm{E}$ and beta carotene for agerelated cataract and vision loss: AREDS report no. 9. Arch Ophthalmol. 2001;119:1439-1452.

6. Van Leeuwen R, Klaver CC, Vingerling JR, et al. The risk and natural course of age-related maculopathy: follow-up at 61/2 years in the Rotterdam Study. Arch Ophthalmol. 2003;121:519-526.

7. Evans JR. Risk factors for age-related macular degeneration. Prog Retinal Eye Res. 2001;20:227-253.

8. Klein R, Klein BE, Jensen SC, Cruickshanks KJ. The relationship of ocular factors to the incidence and progression of age-related maculopathy. Arch Ophthalmol. 1998;116:506-513.
9. van der Schaft TL, Mooy CM, de Bruijn WC, et al. Increased prevalence of disciform macular degeneration after cataract extraction with implantation of an intraocular lens. Br J Opbthalmol. 1994; $78: 441-445$.

10. Maltzman BA, Mulvihill MN, Greenbaum A. Senile macular degeneration and risk factors: a case-control study. Ann Opbthalmol. 1979;11:1197-201.

11. Hyman LG, Lilienfeld AM, Ferris FL III, Fine SL. Senile macular degeneration: a case-control study. Am J Epidemiol. 1983;118: 213-227.

12. Sandberg MA, Tolentino MJ, Miller S, et al. Hyperopia and neovascularization in age-related macular degeneration. Ophthalmology. 1993;100:1009-1013.

13. Age-Related Eye Disease Study Research Group. Risk factors associated with age-related macular degeneration: a case-control study in the age-related eye disease study: age-related eye disease study report number 3. Opbthalmology. 2000;107:2224-2232.

14. The Eye Disease Case-Control Study Group. Risk factors for neovascular age-related macular degeneration. Arch Ophthalmol. 1992;110:1701-1708.

15. Wang JJ, Mitchell P, Smith W. Refractive error and age-related maculopathy: the Blue Mountains Eye Study. Invest Ophthalmol Vis Sci. 1998;39:2167-2171.

16. Wong TY, Klein R, Klein BE, Tomany SC. Refractive errors and 10-year incidence of age-related maculopathy. Invest Ophthalmol Vis Sci. 2002;43:2869-2873.

17. Hofman A, Grobbee DE, de Jong PTVM, van den Ouweland FA. Determinants of disease and disability in the elderly: the Rotterdam Elderly Study. Eur J Epidemiol. 1991;7:403-422.

18. Vingerling JR, Dielemans I, Bots ML, et al. Age-related macular degeneration is associated with atherosclerosis: The Rotterdam Study. Am J Epidemiol. 1995;142:404-409.

19. Bird AC, Bressler NM, Bressler SB, et al. An international classification and grading system for age-related maculopathy and agerelated macular degeneration. The International ARM Epidemiological Study Group. Surv Ophthalmol. 1995;39:367-374.

20. Boker T, Fang T, Steinmetz R. Refractive error and choroidal perfusion characteristics in patients with choroidal neovascularization and age-related macular degeneration. Ger J Opbthalmol. 1993;2:10-13.

21. Friedman E, Ivry M, Ebert E, et al. Increased scleral rigidity and age-related macular degeneration. Ophthalmology. 1989;96:104108 .

22. Friedman E. A hemodynamic model of the pathogenesis of agerelated macular degeneration. Am J Ophthalmol. 1997;124:677682.

23. Ramrattan RS, van der Schaft TL, Mooy CM, et al. Morphometric analysis of Bruch's membrane, the choriocapillaris, and the choroid in aging. Invest Ophthalmol Vis Sci. 1994;35:2857-2864.

24. Kiel JW, Reiner AJ. Morphometric analysis of the choroid, Bruch's membrane, and retinal pigment epithelium in eyes with age-related macular degeneration. Invest Ophthalmol Vis Sci. 1997;38: $1290-1292$

25. Berger JW, Fine SL, Maguire MG. Age-Related Macular Degeneration. St. Louis: Mosby, 1999;xiii, 463.

26. Ciulla TA. Evolving pathophysiological paradigms for age related macular degeneration. Br J Opbthalmol. 2001;85:510-512. 